PROCEEDINGS OF THE

AMERICAN MATHEMATICAL SOCIETY

Volume 42, Number 1, January 1974

\title{
FIXED POINTS OF CERTAIN SELF MAPS ON AN INTERVAL
}

\author{
CHI SONG WONG ${ }^{1}$
}

Abstract. Let $T$ be a self map on a bounded interval $[a, b]$ with $a, b \in T([a, b])$. Suppose that for any $x, y$ in $[a, b]$,

$$
|T(x)-T(y)| \leqq \frac{1}{2}(|x-T(x)|+|y-T(y)|) .
$$

It is shown without the continuity of $T$ that the midpoint of $[a, b]$ is a fixed point of $T$. A nontrivial example is given.

\section{Main theorem.}

THEOREM. Let $T$ be a self map on a bounded closed interval $[a, b]$ with $a, b \in T([a, b])$. Suppose that for all $x, y$ in $[a, b]$,

$$
|T(x)-T(y)| \leqq(|x-T(x)|+|y-T(y)|) / 2 .
$$

Then the midpoint of $[a, b]$ is the unique fixed point of $T$.

ProOF. Since $b-a$ is uniquely maximal for $|x-y|, T(a)=b$ and $T(b)=a$. Let $c$ be the midpoint $(a+b) / 2$ of $[a, b]$. Then $|T(b)-T(c)|$ and $|T(a)-T(c)|$ are each equal to $|a-c|=|b-c|=(b-a) / 2$. Hence $T(c)=c$. From (1), $c$ is the unique fixed point of $T$.

We note here that if $T$ is a continuous self map on a bounded closed interval $Y=[a, b]$ which satisfies (1), then $X=\bigcap_{i=1}^{\infty} T^{i}(Y)$ is nonempty, compact and connected, and $T(X)=X$; hence the midpoint of $X$ is a fixed point of $T$. Comparing this result with that of $\mathrm{R}$. L. Franks and R. P. Marzec [1], we remark here that for any $x$ in $Y$ and for any $t$ in $(0,1),\left\{T_{t}^{n}(x)\right\}$ converges to a fixed point of $T$, where $T_{t}(z)=(1-t) z+t T(z)$ for all $z$ in $Y$. This result was proved in [2] with a much more general setting.

2. Examples. Let $T$ be a function on a bounded interval $X$ into the real line. $T$ is nonexpanding at $x$ in $X$ if $|T(x)-T(y)| \leqq|x-y|$ for all $y$ in $X . T$ is nonexpanding if it is nonexpanding at every point in $X$. If

Received by the editors November 10, 1972 and, in revised form, April 10, 1973. AMS (MOS) subject classifications (1970). Primary 47H10; Secondary 54H25.

${ }^{1}$ This research was partially supported by the National Research Council of Canada Grant A8518 and a grant from the Canadian Mathematical Congress; it was prepared at the Summer Research Institute, Department of Mathematics, University of Alberta. The author wishes to thank the referee for his suggestions.

(c) American Mathematical Society 1974 
$T$ satisfies the conditions of our main theorem, then $T$ is nonexpanding at $x=(a+b) / 2$ and for all $x$ in $[a, b],\left|T^{2}(x)-T(x)\right| \leqq|T(x)-x|$. These are key points in constructing examples.

EXAmple 1. Let $T$ be the self map on $[0,1]$ defined by

$$
T(0)=1, \quad T(1)=0, \quad T(x)=\frac{1}{2}, \text { for all } x \text { in }(0,1) .
$$

Then $T$ is nonexpanding only at $x=\frac{1}{2}$ and satisfies the conditions of our main theorem. Although $T$ is not nonexpanding at any $x$ in $(0,1)$ other than $\frac{1}{2}, T$, restricted to $(0,1)$, is a nonexpanding self map on $(0,1)$.

When $T$ is required to be continuous, the example cannot be so trivial. Indeed, if $T$ is a continuous self map on $[0,1]$ and if $0,1 \in T([0,1])$, then by connectedness of $T([0,1]), T([0,1])=[0,1]$.

EXAMPLE 2. Let $T$ be the self map on $[0,1]$ defined by

$$
\begin{array}{ll}
T(x)=1-2 x+2 x^{2} & \text { if } x \leqq \frac{1}{2}, \\
T(x)=2 x(1-x) & \text { if } x>\frac{1}{2} .
\end{array}
$$

Then: (a) $T$ is a bijective continuous self map on $[0,1]$ and therefore is a homeomorphism of $[0,1]$ onto $[0,1]$. (b) $T$ is differentiable on $(0,1)$ and $\left|T^{\prime}(x)\right| \leqq 1$ for all $x$ in $\left[\frac{1}{4}, \frac{3}{4}\right]$. So by the mean value theorem, $|T(x)-T(y)| \leqq|x-y|$ for all $x, y$ in $\left[\frac{1}{4}, \frac{3}{4}\right]$. However. $T$ is nonexpanding only at $x=\frac{1}{2}$ and $T$, restricted to $\left[\frac{1}{4}, \frac{3}{4}\right]$, is nonexpanding but is not a self map on $\left[\frac{1}{4}, \frac{3}{4}\right]$. (c) $T$ satisfies the conditions of our main theorem. (d) Let $t \in\left(0, \frac{1}{3}\right)$. Let $T_{t}$ be the self map on $[0,1]$ defined by

$$
T_{t}(z)=(1-t) z+t T(z), \quad z \in[0,1] .
$$

Then $x=\frac{1}{2}$ is a fixed point of $T_{t}$. However, $T_{t}$ does not satisfy the condition (1) in our main theorem:

$$
\left|T_{t}(0)-T_{t}\left(\frac{1}{2}\right)\right|=\frac{1}{2}-t
$$

and

$$
\left(\left|0-T_{t}(0)\right|+\left|\frac{1}{2}-T_{t}\left(\frac{1}{2}\right)\right|\right) / 2=t / 2<\frac{1}{2}-t .
$$

The above argument and therefore conclusions hold for any $T$ which satisfies the conditions of our main theorem with $a=0$ and $b=1$.

\section{REFERENCES}

1. R. L. Franks and R. P. Marzec, A theorem on mean-value iterations, Proc. Amer. Math. Soc. 30 (1971), 324-326. MR 43 \#6375.

2. Chi Song Wong, Approximations to fixed points of generalized nonexpansive mappings (to appear).

Department of Mathematics, University of Windsor, Windsor, Ontario, CANADA 